\title{
Definability in the embeddability ordering of finite directed graphs
}

\author{
Ádám Kunos
}

Received: date / Accepted: date

\begin{abstract}
A directed graph $G \in \mathscr{D}$ is said to be embeddable into $G^{\prime} \in \mathscr{D}$ if there exists an injective graph homomorphism $\varphi: G \rightarrow G^{\prime}$. We consider the embeddability ordering $(\mathscr{D}, \leq)$ of finite directed graphs, and prove that for every $G \in \mathscr{D}$ the set $\left\{G, G^{T}\right\}$ is definable by first-order formulas in the partially ordered set $(\mathscr{D}, \leq)$, where $G^{T}$ denotes the transpose of $G$. We also prove that the automorphism group of $(\mathscr{D}, \leq)$ is isomorphic to $\mathbb{Z}_{2}$.
\end{abstract}

Keywords First-order definability · Directed graph · Embeddability ordering

\section{Introduction}

In 2009-2010 J. Ježek and R. McKenzie published a series of papers [1-4] in which they have examined (among other things) the first-order definability in the substructure orderings of finite mathematical structures with a given type and determined the automorphism group of these orderings. They considered finite semilattices [1], ordered sets [2], distributive lattices [3] and lattices [4].

In this paper we consider (the isomorphism types) of finite directed graphs. Let us consider a nonempty set $V$ and a binary relation $E \subseteq V^{2}$. We call the pair $G=(V, E)$ a directed graph or just digraph. The elements of $V(=V(G))$ and $E(=E(G))$ are called the vertices and edges of $G$, respectively. The directed graph $G^{T}:=\left(V, E^{-1}\right)$ is called the transpose of $G$, where $E^{-1}$ denotes the inverse relation of $E$. A directed graph is finite if the number of its vertices is finite. In the papers [1-4] the authors have investigated substructure orderings, meaning that $H \leq H^{\prime}$ if and only if $H$ is isomorphic to a substructure of $H^{\prime}$. Differently, we investigate the embeddability ordering, namely

This research was realized in the frames of TÁMOP 4.2.4. A/2-11-1-2012-0001 "National Excellence Program-Elaborating and operating an inland student and researcher personal support system" The project was subsidized by the European Union and co-financed by the European Social Fund.

Ádám Kunos

University of Szeged, Bolyai Institute, Szeged, Aradi vértanúk tere 1, HUNGARY 6720

E-mail: kunosadam@gmail.com 
we say that the directed graph $G$ is embeddable into $G^{\prime}$ if there exists an injective map $\varphi: V(G) \rightarrow V\left(G^{\prime}\right)$ such that $\left(v_{1}, v_{2}\right) \in E(G)$ implies $\left(\varphi\left(v_{1}\right), \varphi\left(v_{2}\right)\right) \in E\left(G^{\prime}\right)$. It is obvious that isomorphic digraphs are indistinguishable in terms of embeddability. So from now on by a given digraph $G$ we always mean its isomorphism type, for we intend to work with embeddability. Let $\mathscr{D}$ denote the set of isomorphism types of finite digraphs. For $G, G^{\prime} \in \mathscr{D}$, by $G \leq G^{\prime}$ we mean that $G$ is embeddable into $G^{\prime}$. It is easy to verify that $(\mathscr{D}, \leq)$ is a partially ordered set. It is also easy to see that the map $G \mapsto G^{T}(G \in \mathscr{D})$ is a non-trivial automorphism of the poset $(\mathscr{D}, \leq)$. We will prove that there is no other non-trivial automorphism of $(\mathscr{D}, \leq)$.

Let $(\mathscr{A}, \leq)$ be an arbitrary poset. An $n$-ary relation $R$ is said to be definable in $(\mathscr{A}, \leq)$ if there exists a first-order formula $\Psi\left(x_{1}, x_{2}, \ldots, x_{n}\right)$ with free variables $x_{1}, x_{2}, \ldots, x_{n}$ in the language of partially ordered sets such that for any $a_{1}, a_{2}, \ldots, a_{n} \in$ $\mathscr{A}, \Psi\left(a_{1}, a_{2}, \ldots, a_{n}\right)$ holds in $(\mathscr{A}, \leq)$ if and only if $\left(a_{1}, a_{2}, \ldots, a_{n}\right) \in R$. A subset of $\mathscr{A}$ is definable if it is definable as a unary relation. An element $a \in \mathscr{A}$ is said to be definable if the set $\{a\}$ is definable.

In the poset $(\mathscr{D}, \leq)$ let $G \prec G^{\prime}$ denote that $G^{\prime}$ covers $G$. Obviously $\prec$ is a definable relation in $(\mathscr{D}, \leq)$. It is easy to see that if there exists an automorphism of an arbitrary poset $(\mathscr{A}, \leq)$ that maps the element $a \in \mathscr{A}$ to $b \in \mathscr{A}$ then $a$ and $b$ are indistinguishable in $(\mathscr{A}, \leq)$ with first-order formulas. This tells us, considering the fact that $G \rightarrow G^{T}$ is a non-trivial automorphism of $(\mathscr{D}, \leq)$, that the "best" we can prove is that the set $\tilde{G}:=\left\{G, G^{T}\right\}$ is definable for every $G \in \mathscr{D}$. We prove this in the next section.

\section{The definability of the sets $\left\{G, G^{T}\right\}$}

Definition 2.1 Let us introduce the following digraphs:

$E_{1}: V\left(E_{1}\right)=\left\{v_{1}\right\}, E\left(E_{1}\right)=\emptyset$,

$L_{1}: V\left(L_{1}\right)=\left\{v_{1}\right\}, E\left(L_{1}\right)=\left\{\left(v_{1}, v_{1}\right)\right\}$,

$E_{2}: V\left(E_{2}\right)=\left\{v_{1}, v_{2}\right\}, E\left(E_{2}\right)=\emptyset$, and

$I_{2}: V\left(I_{2}\right)=\left\{v_{1}, v_{2}\right\}, E\left(I_{2}\right)=\left\{\left(v_{1}, v_{2}\right)\right\}$.

Lemma 2.2 The digraphs $E_{1}, L_{1}, E_{2}, I_{2}$ are definable.

Proof $E_{1}$ is the unique digraph $X \in \mathscr{D}$ for which $X \leq G$ holds for every $G \in \mathscr{D}$. There are two elements covering $E_{1}$, namely $L_{1}\left(=L_{1}^{T}\right)$ and $E_{2}\left(=E_{2}^{T}\right)$, hence the set $\left\{L_{1}, E_{2}\right\}$ is definable. In this set only $L_{1}$ has a unique cover so $L_{1}$ and $E_{2}$ are distinguishable, which implies that $\tilde{L}_{1}=\left\{L_{1}\right\}$ is definable and so is $\tilde{E}_{2}=\left\{E_{2}\right\} . I_{2}$ is the unique element among the covers of $E_{2}$ which has 4 covers and does not cover $L_{1}$ (see Fig. 1).

Definition 2.3 For a positive integer $n$, we say that $G \in \mathscr{D}$ is on level $n$ if $|V(G)|+$ $|E(G)|=n$. Let $\mathscr{S}_{n}$ denote the set of all digraphs on level $n$.

It is an easy observation that if $G \prec G^{\prime}$ then $G^{\prime}$ is exactly one level above $G$, so our definition corresponds to the levels of the Hasse diagram of $(\mathscr{D}, \leq)$ (see Fig. 1).

Lemma 2.4 The set $\mathscr{S}_{n}$ is definable for every positive integer $n$. 


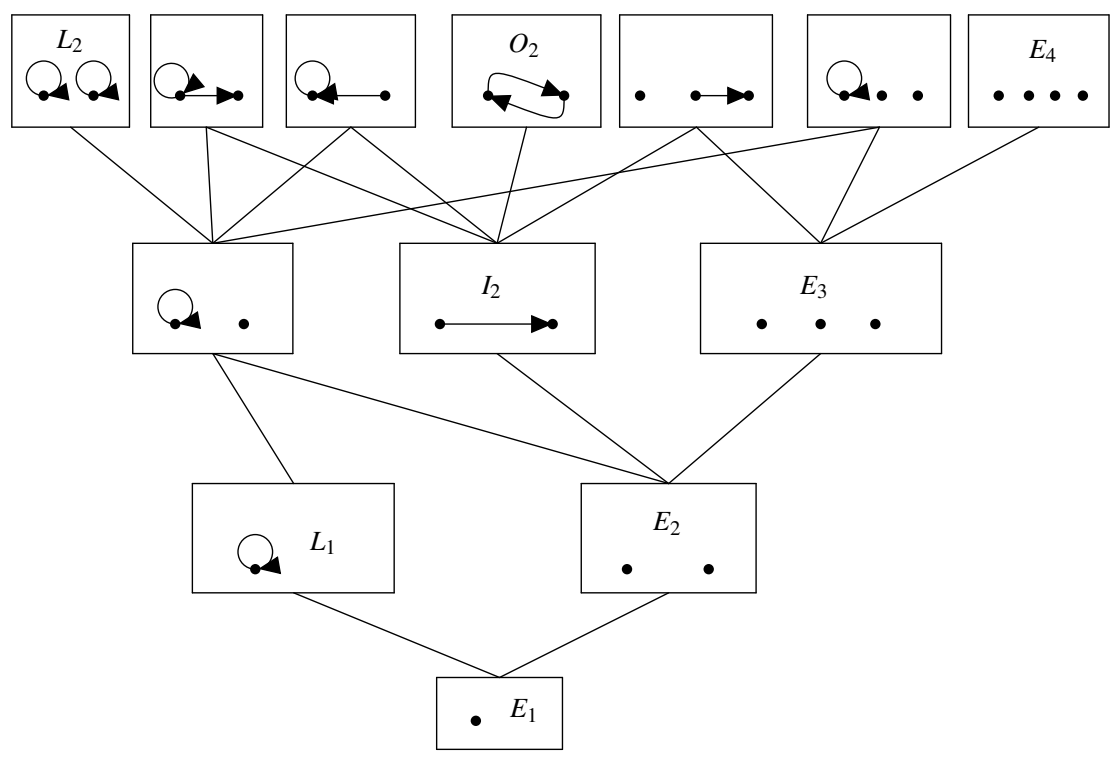

Fig. 1 The "bottom" part of the Hasse diagram of $(\mathscr{D}, \leq)$.

Proof $\mathscr{S}_{1}$ was already defined in Lemma 2.2. $\mathscr{S}_{n+1}$ can be defined recursively: $\mathscr{S}_{n+1}$ is the set of digraphs $X \in \mathscr{D}$ for which $Z \prec X$ holds for some $Z \in \mathscr{S}_{n}$.

Definition 2.5 We say that the digraph $X$ is $n$ level under $Z$ if there exist $Z_{1}, \ldots, Z_{n} \in$ $\mathscr{D}$ such that $Z \succ Z_{1} \succ \cdots \succ Z_{n}=X$. Similarly, we say that the digraph $X$ is $n$ level above $Y$ if there exist $Y_{1}, \ldots, Y_{n} \in \mathscr{D}$ such that $Y \prec Y_{1} \prec \cdots \prec Y_{n}=X$.

Definition 2.6 Let $E_{n}(n=1,2, \ldots)$ be the "empty" digraph with $n$ vertices: $V\left(E_{n}\right)=$ $\left\{v_{1}, v_{2}, \ldots, v_{n}\right\}, E\left(E_{n}\right)=\emptyset$. Let $F_{n}(n=1,2, \ldots)$ be the "full" digraph with $n$ vertices: $V\left(F_{n}\right)=\left\{v_{1}, v_{2}, \ldots, v_{n}\right\}, E\left(F_{n}\right)=V\left(F_{n}\right)^{2}$. Let $\mathscr{T}_{n}=\{G \in \mathscr{D}:|V(G)|=n\}$ be the set of all digraphs having $n$ vertices.

Lemma 2.7 The digraphs $E_{n}, F_{n}$ and the set $\mathscr{T}_{n}$ are definable for every positive integer $n$.

Proof The set $\mathscr{I}=\left\{E_{n}: n \in\{1,2, \ldots\}\right\}$ is definable, because its elements are those digraphs $X \in \mathscr{D}$ for which $L_{1} \not \leq X$ and $I_{2} \not \leq X$. In $\mathscr{I}$ we have $E_{1} \prec E_{2} \prec E_{3} \prec \ldots$, therefore it is easy to see that $E_{n}\left(=E_{n}^{T}\right)$ is definable for every $n$. $\mathscr{T}_{n}$ contains exactly those digraphs $X \in \mathscr{D}$ for which $E_{n} \leq X$ and $E_{n+1} \not \leq X$, so it is definable. $F_{n}$ is the digraph $X \in \mathscr{D}$ which has $n$ vertices and $X \prec Z$ implies that $Z$ has $n+1$ vertices.

Definition 2.8 Let us set notations for the following digraphs (see Fig. 2): $O_{2}: V\left(O_{2}\right)=\left\{v_{1}, v_{2}\right\}, E\left(O_{2}\right)=\left\{\left(v_{1}, v_{2}\right),\left(v_{2}, v_{1}\right)\right\}$, 


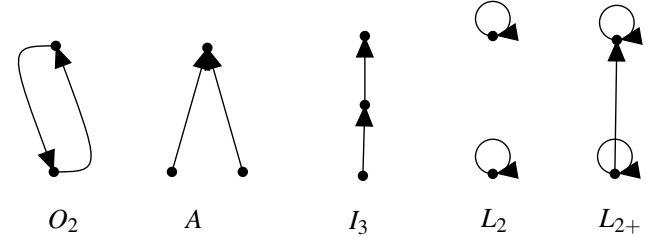

Fig. 2 The digraphs $O_{2}, A, I_{3}, L_{2}, L_{2+}$.

$A: V(A)=\left\{v_{1}, v_{2}, v_{3}\right\}, E(A)=\left\{\left(v_{1}, v_{3}\right),\left(v_{2}, v_{3}\right)\right\}$,

$I_{3}: V\left(I_{3}\right)=\left\{v_{1}, v_{2}, v_{3}\right\}, E\left(I_{3}\right)=\left\{\left(v_{1}, v_{2}\right),\left(v_{2}, v_{3}\right)\right\}$,

$L_{2}: V\left(L_{2}\right)=\left\{v_{1}, v_{2}\right\}, E\left(L_{2}\right)=\left\{\left(v_{1}, v_{1}\right),\left(v_{2}, v_{2}\right)\right\}$, and

$L_{2+}: V\left(L_{2+}\right)=\left\{v_{1}, v_{2}\right\}, E\left(L_{2+}\right)=\left\{\left(v_{1}, v_{1}\right),\left(v_{2}, v_{2}\right),\left(v_{1}, v_{2}\right)\right\}$.

Lemma 2.9 The digraphs $\mathrm{O}_{2}, I_{3}, L_{2}, L_{2}+$ and the set $\tilde{A}$ are definable.

Proof $O_{2}\left(=O_{2}^{T}\right)$ is the maximal digraph $X$ that has 2 vertices and $L_{1} \not \leq X$.

Let $G \in \mathscr{D}$ be the following digraph: $V(G)=\left\{v_{1}, v_{2}, v_{3}\right\}, E(G)=\left\{\left(v_{1}, v_{2}\right)\right\}$. Then $G\left(=G^{T}\right)$ is definable since it is the only element covering both $I_{2}$ and $E_{3}$.

Now the set $\tilde{A} \cup \tilde{I}_{3}$ turns out to be definable, for it contains exactly those digraphs $X \in \mathscr{D}$ for which $G \prec X, O_{2} \not \leq X, E_{4} \not \leq X$ and $L_{1} \not \leq X$ hold. Now $\tilde{I}_{3}=\left\{I_{3}\right\}$ is the unique digraph $X \in \tilde{A} \cup \tilde{I}_{3}$ for which there exists $X \prec Z$ such that $W \prec Z$ implies $X=W$ (with the notation to be introduced in Definition 2.10, $Z=O_{3}$ ), meaning $Z$ covers only $X$. From this we also get that $\tilde{A}$ is definable because $\tilde{A}=\left(\tilde{A} \cup \tilde{I}_{3}\right) \backslash \tilde{I}_{3}$.

The digraph $L_{2}$ is the maximal $X \in \mathscr{D}$ that has 2 vertices and for which $I_{2} \not \leq X$. Finally, the digraph $L_{2+}$ is the only $X \in \mathscr{D}$ having 2 vertices, being on level 5 for which $L_{2} \leq X$.

Definition 2.10 Let $I_{n}, O_{n}, L_{n}(n=2,3, \ldots)$ denote the following digraphs:

$$
\begin{gathered}
V\left(I_{n}\right)=V\left(O_{n}\right)=V\left(L_{n}\right)=\left\{v_{1}, v_{2}, \ldots, v_{n}\right\}, \\
E\left(I_{n}\right)=\left\{\left(v_{1}, v_{2}\right),\left(v_{2}, v_{3}\right), \ldots,\left(v_{n-1}, v_{n}\right)\right\}, \\
E\left(O_{n}\right)=E\left(I_{n}\right) \cup\left\{\left(v_{n}, v_{1}\right)\right\}=\left\{\left(v_{1}, v_{2}\right),\left(v_{2}, v_{3}\right), \ldots,\left(v_{n-1}, v_{n}\right),\left(v_{n}, v_{1}\right)\right\}, \text { and } \\
E\left(L_{n}\right)=\left\{\left(v_{1}, v_{1}\right),\left(v_{2}, v_{2}\right), \ldots,\left(v_{n}, v_{n}\right)\right\} .
\end{gathered}
$$

Lemma 2.11 The digraphs $L_{n}, O_{n}, I_{n}(n=2,3, \ldots)$ are definable.

Proof $L_{n}\left(=L_{n}^{T}\right)$ is the unique digraph $X$ on level $2 n$ that has $n$ vertices and for which $I_{2} \not \leq X$.

We define the digraphs $O_{n}\left(=O_{n}^{T}\right)$ and $I_{n}\left(=I_{n}^{T}\right)$ together, recursively. $O_{2}$ and $I_{2}$ were already defined in Lemma 2.9 and Lemma 2.2, respectively. Suppose that $O_{2}, I_{2}, \ldots$, $O_{n}, I_{n}$ have already been defined. Then $O_{n+1}$ is the unique digraph $X$ that:

- has $n+1$ vertices,

$-I_{n} \leq X, L_{1} \not \leq X, O_{2} \not \leq X, O_{n} \not \leq X$, 

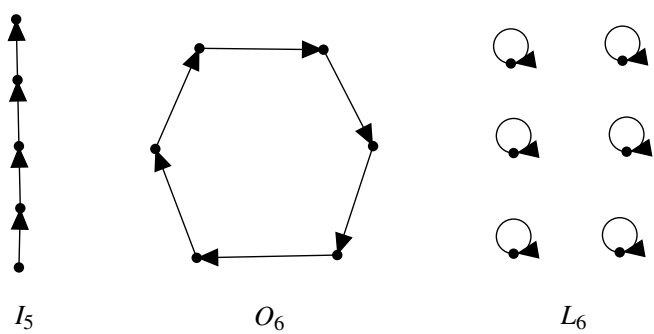

Fig. 3 The digraphs $I_{5}, O_{6}$ and $I_{6}$.

- is on level $2 n+2$, and

- there exists no $Z \in \tilde{A}$ for which $Z \leq X$.

Finally, the digraph $I_{n+1}$ is the only element $X$ for which $X \prec O_{n+1}$.

Definition 2.12 Let $G$ be an arbitrary finite directed graph with no loops. Let $L(G)$ denote the digraph that we get from $G$ by adding loops to every vertex. For a set $\mathscr{G} \subseteq \mathscr{D}$ of finite digraphs with no loops let $\mathscr{L}(\mathscr{G}):=\{L(G): G \in \mathscr{G}\}$.

Definition 2.13 For an arbitrary $G \in \mathscr{D}$ let $M(G)$ denote the digraph we get by leaving all the loops out from $G$. For a set $\mathscr{G} \subseteq \mathscr{D}$ of finite digraphs put $\mathscr{M}(\mathscr{G}):=\{M(G)$ : $G \in \mathscr{G}\}$.

Lemma 2.14 Let $\mathscr{G} \subseteq \mathscr{D}$ be a definable set of finite digraphs with no loops. Then the set $\mathscr{L}(\mathscr{G})$ is definable.

Proof We define the binary relation

$$
\alpha=\{(G, M(G)): G \in \mathscr{D}\}
$$

as the set of pairs $(X, Y) \in \mathscr{D}^{2}$ for which $Y$ is the maximal digraph with $Y \leq X$ and $L_{1} \not \leq Y$. Now $\mathscr{L}(\mathscr{G})$ is the set of those digraphs $X$ for which there exists $Y \in \mathscr{G}$ such that $X$ is the maximal digraph with $(X, Y) \in \alpha$.

Lemma 2.15 Let $\mathscr{G} \subseteq \mathscr{D}$ be a definable set of finite digraphs. Then the set $\mathscr{M}(\mathscr{G})$ is definable.

Proof The set $\mathscr{M}(\mathscr{G})$ is definable as the set of those digraphs $X$ for which there exists $Y \in \mathscr{G}$ such that $X$ is the maximal digraph with $X \leq Y$ and $L_{1} \not \leq X$.

Definition 2.16 Let $O_{n, L}$ be the following digraph (see Fig. 4): $V\left(O_{n, L}\right)=\left\{v_{1}, v_{2}, \ldots, v_{n}\right\}$, $E\left(O_{n, L}\right)=E\left(O_{n}\right) \cup\left\{\left(v_{1}, v_{1}\right)\right\}$, which means that

$$
E\left(O_{n, L}\right)=\left\{\left(v_{1}, v_{1}\right),\left(v_{1}, v_{2}\right),\left(v_{2}, v_{3}\right), \ldots,\left(v_{n-1}, v_{n}\right),\left(v_{n}, v_{1}\right)\right\} .
$$

Lemma 2.17 The digraphs $O_{n, L}(n=2,3, \ldots)$ are definable. 


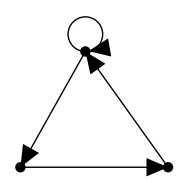

Fig. 4 The digraph $O_{3, L}$
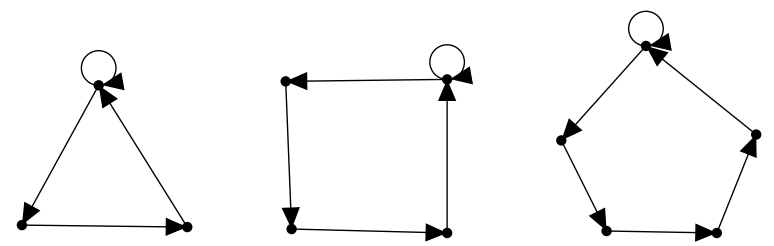

Fig. 5 The digraph $O_{L}(3,4,5)$.

Proof $O_{n, L}\left(=O_{n, L}^{T}\right)$ is the unique digraph $X$ on level $2 n+1$ for which $O_{n} \leq X$ and $L_{1} \leq X$.

Definition 2.18 For arbitrary finite directed graphs $G_{1}, G_{2}, \ldots, G_{n}$ let us denote their disjoint uniun by $G_{1} \dot{\cup} G_{2} \dot{\cup} \ldots \dot{\cup} G_{n}=\dot{\bigcup}_{i=1}^{n} G_{i}$, as usual.

Definition 2.19 Let $n_{1}, n_{2}, \ldots, n_{k}$ be pairwise distinct integers greater than 1 . Let

$$
O\left(n_{1}, n_{2}, \ldots, n_{k}\right)=\bigcup_{i=1}^{k} O_{n_{i}} ; \quad O_{L}\left(n_{1}, n_{2}, \ldots, n_{k}\right)=\bigcup_{i=1}^{k} O_{n_{i}, L}
$$

Lemma 2.20 The digraphs $O\left(n_{1}, n_{2}, \ldots, n_{k}\right)$ and $O_{L}\left(n_{1}, n_{2}, \ldots, n_{k}\right)$ are definable.

Proof $O\left(n_{1}, n_{2}, \ldots, n_{k}\right)\left(=\left(O\left(n_{1}, n_{2}, \ldots, n_{k}\right)\right)^{T}\right)$ is the unique digraph $X$ having $n_{1}+$ $n_{2}+\cdots+n_{k}$ vertices, being on level $2\left(n_{1}+n_{2}+\cdots+n_{k}\right)$ for which $O_{n_{i}} \leq X(i=$ $1,2, \ldots, k)$ and there exists no $Z \in \tilde{A}$ such that $Z \leq X$.

$O_{L}\left(n_{1}, n_{2}, \ldots, n_{k}\right)\left(=\left(O_{L}\left(n_{1}, n_{2}, \ldots, n_{k}\right)\right)^{T}\right)$ is the unique digraph $X$ being on level $2\left(n_{1}+n_{2}+\cdots+n_{k}\right)+k$ for which $O\left(n_{1}, n_{2}, \ldots, n_{k}\right) \leq X, L_{k} \leq X$ and $O_{n_{i}, L} \leq X(i=$ $1,2, \ldots, k)$.

Definition 2.21 Let $i$ and $j$ be different positive integers both bigger than 2. Let us define the digraph $O_{i, j, L 1}$ (see Fig. 6) the following way. Let $V\left(O_{i}\right)=\left\{v_{1}, \ldots, v_{i}\right\}$ and $V\left(O_{j}\right)=\left\{v_{1}^{\prime}, \ldots, v_{j}^{\prime}\right\}$. Now let $V\left(O_{i, j, L 1}\right)=V\left(O_{i} \cup O_{j}\right)$,

$$
E\left(O_{i, j, L 1}\right)=E\left(O_{i} \cup O_{j}\right) \cup\left\{\left(v_{1}, v_{1}\right),\left(v_{1}^{\prime}, v_{1}^{\prime}\right),\left(v_{1}, v_{1}^{\prime}\right)\right\} .
$$

Let $O_{i, j, L 2}: V\left(O_{i, j, L 2}\right)=V\left(O_{i, j, L 1}\right), E\left(O_{i, j, L 2}\right)=E\left(O_{i, j, L 1}\right) \cup\left\{\left(v_{1}^{\prime}, v_{1}\right)\right\}$ and finally let $O_{i, j, 1}=M\left(O_{i, j, L 1}\right), O_{i, j, 2}=M\left(O_{i, j, L 2}\right)$.

In words, $O_{i, j, L 1}$ consists of two circles which are connected by an $L_{2+}$ (see Fig. 6).

Lemma 2.22 Let $i$ and $j$ be different positive integers both bigger than 2. Then the sets $\tilde{O}_{i, j, L 1}, \tilde{O}_{i, j, L 2}, \tilde{O}_{i, j, 1}$ and $\tilde{O}_{i, j, 2}$ are definable. 


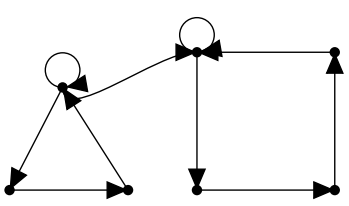

$O_{3,4, L 1}$

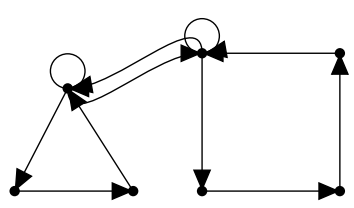

$O_{3,4, L 2}$

Fig. $6 O_{3,4, L 1}, O_{3,4, L 2}$

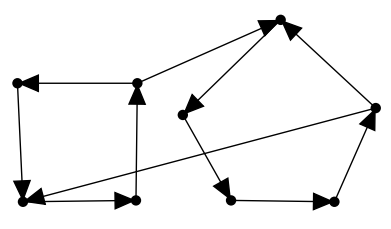

X

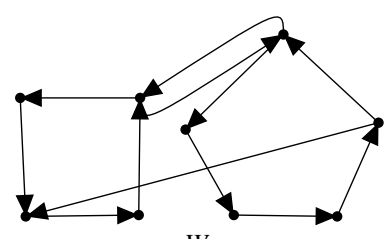

W

Fig. 7 A digraph $X \in \mathscr{O}_{4,5,1}^{+}$and a digraph $W \in \mathscr{O}_{4,5,2}^{+}$.

Proof $\tilde{O}_{i, j, L 1}$ is the set of those digraphs $X$ that:

- have $i+j$ vertices,

- are on level $2(i+j)+3$, and

- $O_{L}(i, j) \leq X, L_{2+} \leq X, L_{3} \not X X$.

$\tilde{O}_{i, j, L 2}$ consists of those digraphs $X$ that:

- are on level $2(i+j)+4$,

- $F_{2} \leq X$, and

- there exists $Z \in \tilde{O}_{i, j, L 1}$ such that $Z \leq X$.

Finally, the definability of the digraphs $\tilde{O}_{i, j, 1}, \tilde{O}_{i, j, 2}$ follows from Lemma 2.15 .

Definition 2.23 Let $\mathscr{O}_{i, j, 1}^{+}$denote the set of those digraphs covering $Z$ in $\mathscr{D}$ which are obtained by adding an edge to $Z$ that is not a loop, where $Z \in \tilde{O}_{i, j, 1}$. Similarly, let $\mathscr{O}_{i, j, 2}^{+}$denote the set of those digraphs covering $Z$ in $\mathscr{D}$ which are obtained by adding an edge to $Z$ that is not a loop, where $Z \in \tilde{O}_{i, j, 2}$. (see Fig. 7).

Lemma 2.24 The sets $\mathscr{O}_{i, j, 1}^{+}, \mathscr{O}_{i, j, 2}^{+}$are definable for every distinct positive integers $i, j$ both bigger than two.

Proof $\mathscr{O}_{i, j, 1}^{+}$consists of those digraphs $X$ for which $Z \prec X$ for some $Z \in \tilde{O}_{i, j, 1}, L_{1} \not \leq X$ and $E_{i+j+1} \not \leq X$. Similarly, $\mathscr{O}_{i, j, 2}^{+}$is the set of those digraphs $X$ for which $Z \prec X$ for some $Z \in \tilde{O}_{i, j, 2}, L_{1} \not \leq X$ and $E_{i+j+1} \not \leq X$.

Definition 2.25 For a finite directed graph $G \in \mathscr{D}$, let $\mathscr{A}(G)$ be the set of those digraphs that can be obtained from $G$ by reversing some edges. 
Definition 2.26 Let $u, v, w$ be 3 distinct vertices of a directed graph $G \in \mathscr{D}$. Let $\left.G\right|_{\{u, v, w\}}$ denote the digraph induced by the vertices $u, v, w$. Let $[u, v, w]_{G}$ denote the digraph for which $V\left([u, v, w]_{G}\right):=V\left(\left.G\right|_{\{u, v, w\}}\right)$ and

$$
E\left([u, v, w]_{G}\right):=E\left(\left.G\right|_{\{u, v, w\}}\right) \cap\{(u, v),(v, u),(v, w),(w, v)\} .
$$

Lemma 2.27 Let $G \in \mathscr{D}$ be a weakly connected digraph with vertices $v_{1}, \ldots, v_{n}$ for which $\mathrm{O}_{2} \nless \leq G$. Then

$$
\begin{aligned}
\left\{G, G^{T}\right\}=\{X \in \mathscr{A}(G): & \left(\left[v_{i}, v_{j}, v_{k}\right]_{X}=I_{3}\right) \Leftrightarrow\left(\left[v_{i}, v_{j}, v_{k}\right]_{G}=I_{3}\right), \\
& \left.\left(\left[v_{i}, v_{j}, v_{k}\right]_{X} \in \tilde{A}\right) \Leftrightarrow\left(\left[v_{i}, v_{j}, v_{k}\right]_{G} \in \tilde{A}\right)\right\} .
\end{aligned}
$$

Proof We can suppose that $G$ has an edge because otherwise $G$ must have a single vertex which is a trivial case. We can also suppose that $\left(v_{1}, v_{2}\right) \in E(G)$, for it is only a matter of notation. Let $H$ denote the right-hand side of (2). It is clear that for any $X \in H$, there is exactly one edge between the vertices $v_{1}$ and $v_{2}$ since $H \subseteq \mathscr{A}(G)$ and $O_{2} \not \leq G$. Let us suppose first that for an $X \in H$, similarly to $G,\left(v_{1}, v_{2}\right) \in E(X)$ holds. We claim that $X=G$. Let us consider an arbitrary pair of vertices $v^{\prime}, v^{\prime \prime} \in V(X)$ that are connected by an edge in $X$. Since $G$ is weakly connected there exists a series

$$
v_{1}=w_{1}, v_{2}=w_{2}, w_{3}, \ldots, w_{k-2}, v^{\prime}=w_{k-1}, v^{\prime \prime}=w_{k}
$$

of pairwise distinct vertices such that for all $j \in\{1,2, \ldots, k-1\}$ there is exactly one edge between the edges $w_{j}, w_{j+1}$. Let us consider the neighbouring vertices in this series. We know that $\left(w_{1}, w_{2}\right) \in E(G), E(X)$. Observe that the direction of the edge between the vertices $w_{2}, w_{3}$ in $X$ is determined by the conditions

$$
\begin{aligned}
\left(\left[w_{1}, w_{2}, w_{3}\right]_{X}=I_{2}\right) & \Leftrightarrow\left(\left[w_{1}, w_{2}, w_{3}\right]_{G}=I_{2}\right), \\
\left(\left[w_{1}, w_{2}, w_{3}\right]_{X} \in \tilde{A}\right) & \Leftrightarrow\left(\left[w_{1}, w_{2}, w_{3}\right]_{G} \in \tilde{A}\right),
\end{aligned}
$$

moreover it has the same direction in $X$ as in $G$ and so on, the direction of the edge between the vertices $w_{j}, w_{j+1}$ is determined by the conditions

$$
\begin{aligned}
\left(\left[w_{j-1}, w_{j}, w_{j+1}\right]_{X}=I_{2}\right) & \Leftrightarrow\left(\left[w_{j-1}, w_{j}, w_{j+1}\right]_{G}=I_{2}\right), \\
\left(\left[w_{j-1}, w_{j}, w_{j+1}\right]_{X} \in \tilde{A}\right) & \Leftrightarrow\left(\left[w_{j-1}, w_{j}, w_{j+1}\right]_{G} \in \tilde{A}\right)
\end{aligned}
$$

and it has the same direction in $X$ and $G$. We proved that the direction of the edge connecting vertices $v^{\prime}, v^{\prime \prime}$ is the same in $X$ as in $G$, therefore we proved $X=G$. If we suppose the converse: $\left(v_{2}, v_{1}\right) \in E(X)$ then $X^{T}=G$ by the previous case.

Definition 2.28 For pairwise distinct positive integers $i, j, k$ greater than 2 we define the digraph $O_{i \rightarrow j \rightarrow k}$ (see Fig. 8) the following way. Let $V\left(O_{i}\right)=\left\{v_{1}, v_{2}, \ldots, v_{i}\right\}$, $V\left(O_{j}\right)=\left\{v_{1}^{\prime}, v_{2}^{\prime}, \ldots, v_{j}^{\prime}\right\}$ and $V\left(O_{k}\right)=\left\{v_{1}^{\prime \prime}, v_{2}^{\prime \prime}, \ldots, v_{k}^{\prime \prime}\right\}$. Now let $V\left(O_{i \rightarrow j \rightarrow k}\right)=V\left(O_{i} \cup\right.$ $\left.O_{j} \cup O_{k}\right)$ and

$$
E\left(O_{i \rightarrow j \rightarrow k}\right)=E\left(O_{i} \cup O_{j} \cup O_{k}\right) \cup\left\{\left(v_{1}, v_{1}\right),\left(v_{1}^{\prime}, v_{1}^{\prime}\right),\left(v_{1}^{\prime \prime}, v_{1}^{\prime \prime}\right),\left(v_{1}, v_{1}^{\prime}\right),\left(v_{1}^{\prime}, v_{1}^{\prime \prime}\right)\right\} .
$$

$O_{i \rightarrow j \leftarrow k}$ is defined similarly, by modifying the definition of $O_{i \rightarrow j \rightarrow k}$ naturally by replacing $\left(v_{1}^{\prime}, v_{1}^{\prime \prime}\right)$ with $\left(v_{1}^{\prime \prime}, v_{1}^{\prime}\right)$. 


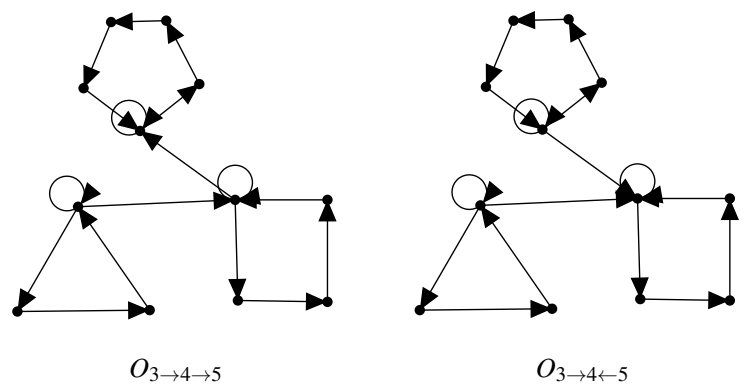

Fig. 8 The digraphs $O_{3 \rightarrow 4 \rightarrow 5}$ and $O_{3 \rightarrow 4 \leftarrow 5}$

In words, $O_{i \rightarrow j \rightarrow k}$ and $O_{i \rightarrow j \leftarrow k}$ consist of three disjoint circles $O_{i}, O_{j}$, $O_{k}$ with one loop on each that are connected in the way $i \rightarrow j \rightarrow k$ and $i \rightarrow j \leftarrow k$ according to the sizes of the circles they are on (see Fig. 8).

Lemma 2.29 Let $i, j, k$ be pairwise distinct positive integers bigger than 2 . Then the sets $\tilde{O}_{i \rightarrow j \rightarrow k}$ and $\tilde{O}_{i \rightarrow j \leftarrow k}$ are definable.

Proof $\tilde{O}_{i \rightarrow j \rightarrow k}$ is the set of those digraphs $X$ that:

- have $i+j+k$ vertices,

- $O_{L}(i, j, k) \leq X$

- are on level $2(i+j+k)+5$,

- there exist $Z \in \tilde{O}_{i, j, L 1}$ and $W \in \tilde{O}_{j, k, L 1}$ such that $Z, W \leq X$, and

$-L\left(I_{3}\right) \leq X$.

We can define $\tilde{O}_{i \rightarrow j \leftarrow k}$ almost the same way as $\tilde{O}_{i \rightarrow j \rightarrow k}$, the difference is that we replace the condition $L\left(I_{3}\right) \leq X$ by: there exists $Z \in \mathscr{L}(\tilde{A})$ for which $Z \leq X$.

Definition 2.30 Let $G$ be a finite directed graph with $n$ vertices and no loops and let $\underline{v}=\left(v_{1}, v_{2}, \ldots, v_{n}\right)$ be a vector containing all the vertices of $G$ in some fixed order. Let us define the digraph $K(G, \underline{v})$ the following way. Let us consider the circles $O_{n+1}, O_{n+2}, \ldots, O_{2 n}$ with $V\left(O_{n+i}\right)=\left\{v_{i, j}: 1 \leq j \leq n+i\right\}$. Now let

$$
\begin{gathered}
V(K(G, \underline{v}))=V\left(\bigcup_{1 \leq i \leq n}^{\cdot} O_{n+i}\right), \\
E(K(G, \underline{v}))=E\left(\bigcup_{1 \leq i \leq n} O_{n+i}\right) \cup\left\{\left(v_{i, 1}, v_{j, 1}\right):\left(v_{i}, v_{j}\right) \in E(G)\right\} \\
\cup\left\{\left(v_{i, 1}, v_{i, 1}\right): 1 \leq i \leq n\right\} .
\end{gathered}
$$

In words we get $K(G, \underline{v})$ from $G$ by adding loops and big, differently sized circles to all the vertices of $G$ (see Fig. 9).

Example 1 Figure 9 shows $K\left(I_{3}, \underline{v}\right)$ as an example (with $\underline{v}=\left(v_{1}, v_{2}, v_{3}\right)$ ). 

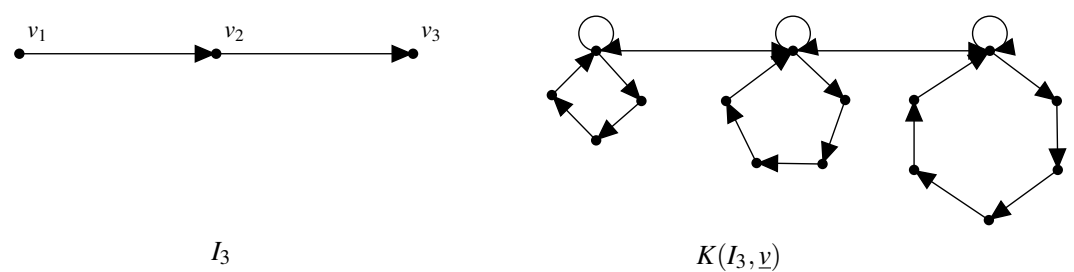

$K\left(I_{3}, \underline{v}\right)$

Fig. $9 I_{3}$ and a corresponding $K\left(I_{3}, \underline{v}\right)$.

This type of graph will be useful because in this graph - thanks to the big circleswe can distinguish between the vertices of $G$ which will allow us to define which pairs of vertices are connected with how many edges, so we will be able to define the set $\mathscr{A}(G)$

Lemma 2.31 For an arbitrary weakly connected finite digraph $G \in \mathscr{D}$ with no loops the set $\tilde{G}=\left\{G, G^{T}\right\}$ is definable.

Proof First we consider all the pairs of vertices that have edges in both directions between them and we clear one of the two edges for each such pair. We may get different digraphs clearing different edges but as for the proof it does not matter which one we take so let us fix one such digraph $G^{\prime}$ with $O_{2} \not \leq G^{\prime}$ for the rest of the proof.

Let $\underline{v}=\left(v_{1}, v_{2}, \ldots, v_{n}\right)$ be a fixed vector of the vertices of $G$. We first define the set

$$
\mathscr{H}_{1}:=\left\{K\left(G^{\prime}, \underline{v}\right), K\left(\left(G^{\prime}\right)^{T}, \underline{v}\right)\right\} .
$$

This set contains exactly those digraphs $X$ that:

(1) have $(n+1)+(n+2)+\cdots+(n+n)\left(=\frac{n(3 n+1)}{2}\right)$ vertices,

(2) $O_{L}(n+1, n+2, \ldots, n+n) \leq X$,

(3) $L_{n+1} \not \leq X$,

(4) if there is no edge between the vertices $v_{i}, v_{j}$ in $G^{\prime}$, then for all $Z \in \tilde{O}_{n+i, n+j, 1}$, $Z \nless \leq X$ holds,

(5) if there is an edge between the vertices $v_{i}, v_{j}$ in $G^{\prime}$ then there is an $Z \in \tilde{O}_{n+i, n+j, L 1}$ for which $Z \leq X$ but for all $W \in \mathscr{O}_{n+i, n+j, 1}^{+}, W \not \leq X$ holds,

(6) if $\left[v_{i}, v_{j}, v_{k}\right]_{G^{\prime}}=I_{3}$, there exists a $Z \in \tilde{O}_{(n+i) \rightarrow(n+j) \rightarrow(n+k)}$ for which $Z \leq X$, and

(7) if $\left[v_{i}, v_{j}, v_{k}\right]_{G^{\prime}} \in \tilde{A}$ there exists a $Z \in \tilde{O}_{(n+i) \rightarrow(n+j) \leftarrow(n+k)}$ for which $Z \leq X$.

The conditions (1)-(3) ensure the structure of "big" circles, determine the number of loops. The conditions (4)-(5) tell how many and what kind of edges are to be drawn between the "big circles". The conditions (1)-(5) define the set

$$
\left\{K(Z, \underline{v}): Z \in \mathscr{A}\left(G^{\prime}\right)\right\}
$$

while the conditions (6)-(7), by Lemma 2.27 , choose the set $\mathscr{H}_{1}$ from it. It is easy to see that the conditions (1)-(5) can similarly define the set

$$
\mathscr{H}_{2}:=\{K(Z, \underline{v}): Z \in \mathscr{A}(G)\}
$$




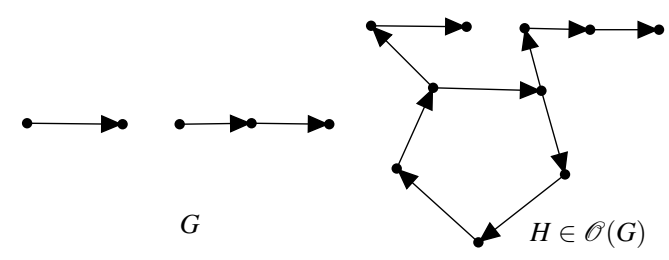

Fig. 10 A digraph $G$ and a corresponding $H \in \mathscr{O}(G)$.

with the following condition added (and writing $G$ instead of $G^{\prime}$ in every other condition, naturally):

(8) if there are edges between the vertices $v_{i}, v_{j}$ in both directions then there exists a digraph $Z \in \tilde{O}_{n+i, n+j, L 2}$ for which $Z \leq X$ but for any $W \in \mathscr{O}_{n+i, n+j, 2}^{+}, W \not \leq X$ holds.

Now the set

$$
\mathscr{H}_{3}:=\left\{K(G, \underline{v}), K\left((G)^{T}, \underline{v}\right)\right\}
$$

can be defined the following way. It consists of those digraphs $X$ that:

(9) $X \in \mathscr{H}_{2}$, and

(10) there exists a digraph $Z \in \mathscr{H}_{1}$ for which $Z \leq X$.

Finally the set $\mathscr{H}_{4}=\left\{L(G), L\left(G^{T}\right)\right\}$ consists of those $X$ that:

(11) have $n$ vertices,

(12) $L_{n} \leq X$,

(13) $X \leq Z$ for some $Z \in \mathscr{H}_{3}$, and

(14) $X$ is maximal with the previous properties.

Finally we can prove the definability of $\tilde{G}$ using Lemma 2.15 , for $\mathscr{M}\left(\mathscr{H}_{4}\right)=\left\{G, G^{T}\right\}$.

Let $G_{1}, G_{2}, \ldots, G_{n}$ be the weakly connected components of an arbitrary $G \in \mathscr{D}$. Let $v_{1} \in G_{1}, v_{2} \in G_{2}, \ldots, v_{n} \in G_{n}$ be arbitrary but fixed vertices. Let $N=|V(G)|$. Let us build the digraph $H$ the following way. We add the vertices $v_{1}^{\prime}, v_{2}^{\prime}, \ldots, v_{N}^{\prime}$ to the digraph $G$ so that they constitute an $O_{N}$ circle. We make this digraph, having $n+1$ weakly connected components, weakly connected by adding the edges $\left(v_{1}^{\prime}, v_{1}\right)$, $\left(v_{2}^{\prime}, v_{2}\right), \ldots,\left(v_{n}^{\prime}, v_{n}\right)$. It is clear that this contruction depends only on the choice of the $v_{i}$ 's.

Definition 2.32 Let us denote by $\mathscr{O}(G)$ the set of all digraphs $H$ that can be created this way.

Example 2 In Figure 10 we can see a digraph $G$ having 2 weakly connected components and an $H \in \mathscr{O}(G)$.

Lemma 2.33 For every finite directed graph $G \in \mathscr{D}$ with no loops the set $\left\{G, G^{T}\right\}$ is definable. 


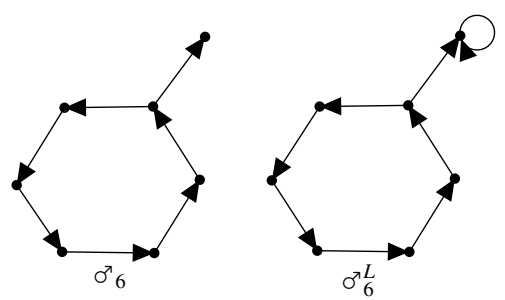

Fig. $11 \sigma^{x}{ }_{6}$ and $\sigma_{6}^{2}$.

Proof We only need to deal with those $G \in \mathscr{D}$ that have more than one weakly independent components, for we have dealt with the other case before. Let us consider a digraph $H \in \mathscr{O}(G)$. Since $H$ is weakly connected and does not have loops, we know that $\tilde{H}$ is definable by Lemma 2.31 . Let $H^{\prime}$ be the digraph that we get from $H$ by adding loops to all the vertices corresponding to $G$ (that do not belong to the "big circle").

Let $|V(G)|=N$. $\tilde{H}^{\prime}$ is the set of those digraphs $X \in \mathscr{D}$ such that

- there exists $Z \in \tilde{H}$ such that $Z$ is $N$ level under $X$, and

- $L_{N} \leq X, O_{N, L} \not X X$.

In $H^{\prime}$ there are loops exactly on those vertices that correspond to $G$ which allows us to define $\mathscr{L}(\tilde{G})$ : it is the set of those digraphs $X$ such that:

- $X \leq Z$ for some $Z \in \tilde{H}^{\prime}$,

- $X$ has $N$ vertices and $L_{N} \leq X$, and

$-X$ is maximal with the previous properties.

We are done since $\tilde{G}=\mathscr{M}(\mathscr{L}(\tilde{G}))$ is definable by Lemma 2.15 .

Definition 2.34 Let $\sigma^{7} n$ denote the following digraph (see Fig. 11). Let $V\left(O_{n}\right)=$ $\left\{v_{1}, \ldots, v_{n}\right\}$ and let us define $\sigma^{\pi} n$ with $V\left(\sigma^{\pi}{ }_{n}\right)=V\left(O_{n}\right) \cup\{v\}$ and $E\left(\sigma_{n} n\right)=E\left(O_{n}\right) \cup$ $\left\{\left(v_{1}, v\right)\right\}$. Let $\sigma^{x}{ }_{n}^{L}$ denote the digraph that is obtained from $\sigma^{\top}{ }_{n}$ by adding a loop the following way:

$$
E\left(\sigma^{\top}{ }_{n}^{L}\right)=E\left(\sigma^{\top} n\right) \cup\{(v, v)\}
$$

Since $\sigma_{n}{ }_{n}$ is weakly connected and has no loops, $\tilde{\sigma}_{n}$ is definable by Lemma 2.31 .

Lemma 2.35 The digraphs ${\tilde{\sigma^{2}}}_{n}^{L}(n=2,3, \ldots)$ are definable.

Proof ${\tilde{\sigma^{T}}}_{n}^{L}$ is the set of those digraphs $X$ such that:

- $Z \prec X$ for some $Z \in \tilde{\sigma}_{n}$, and

- $L_{1} \leq X$ and $O_{n, L} \not \leq X$.

Lemma 2.36 For an arbitrary weakly connected finite directed graph $G \in \mathscr{D}$ the set $\left\{G, G^{T}\right\}$ is definable. 


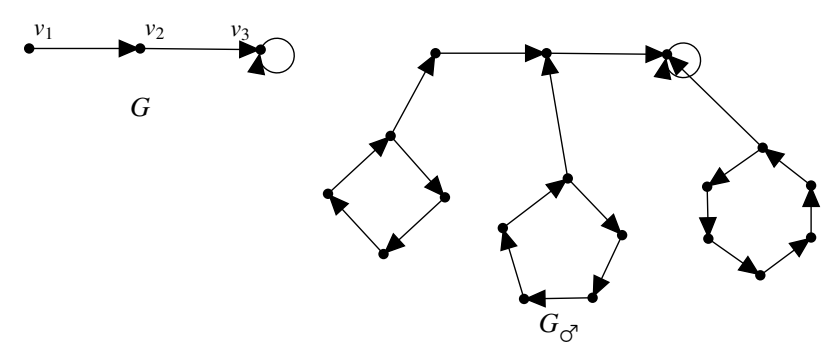

Fig. $12 G_{\sigma^{T}}$ for a given $G$ with a given order of vertices.

Proof Let $v_{1}, v_{2}, \ldots, v_{n}$ be the vertices of $G$. Let us consider the circles $\left\{O_{n+i}\right\}_{i=1}^{n}$ so that $V\left(O_{n+i}\right)=\left\{v_{n+i, j}: 1 \leq j \leq n+i\right\}$. We define the digraph $G_{\sigma^{\pi}}$ with

$$
\begin{gathered}
V\left(G_{\sigma^{\pi}}\right)=V\left(G \dot{\cup}\left(\bigcup_{i=1}^{n} O_{n+i}\right)\right), \text { and } \\
E\left(G_{\sigma^{x}}\right)=E\left(G \dot{\cup}\left(\bigcup_{i=1}^{n} O_{n+i}\right)\right) \cup\left\{\left(v_{n+i, 1}, v_{i}\right): 1 \leq i \leq n\right\} .
\end{gathered}
$$

In words, we add big circles to $G$ and connect them to the vertices of $G$ with edges pointing in $G$ 's direction. $\left(G_{O^{x}}\right.$ depends on the order of $v_{1}, v_{2}, \ldots, v_{n}$. We do not emphasize this in the notation because we need this structure only once and here it is enough to think of any fixed order of the vertices.) An example of the construction is shown in Figure 12.

Since $M\left(G_{\sigma^{7}}\right)$ is weakly connected and has no loops, the set $\mathscr{M}\left(\tilde{G}_{\sigma^{7}}\right)$ is definable by Lemma 2.31. We can suppose that $G$ has at least one loop because we dealt with the other case in Lemma 2.31. Let $v_{i_{1}}, v_{i_{2}}, \ldots, v_{i_{k}}$ be the list of vertices of $G$ with loops. $\tilde{G}_{\sigma^{x}}$ is now the set of those digraphs $X$ such that:

- $X$ is $k$ level above some $Z \in \mathscr{M}\left(\tilde{G}_{\sigma^{7}}\right)$, and

- for every $1 \leq l \leq k$ there exists $Z \in{\tilde{\sigma^{7}}}_{n+i_{l}}^{L}$ for which $Z \leq X$.

Let $G_{\sigma^{7}}^{\prime}=G \dot{\cup}\left(\dot{\cup}_{i=1}^{n} O_{n+i}\right)$. Then $\tilde{G}_{\sigma^{7}}^{\prime}$ is the set of those $X$ that:

- are $n$ level under $Z$ for some $Z \in \tilde{G}_{\sigma^{7}}$,

- $Z \in \tilde{\sigma}_{n+i}$ implies $Z \not \leq X$ for every $1 \leq i \leq n$, and

- $O_{n+i} \leq X$ for every $1 \leq i \leq n$.

Finally, $\tilde{G}$ consists of those $X$ that:

- $X \leq Z$ for some $Z \in \tilde{G}_{O^{\prime \prime}}^{\prime}$,

- have $n$ vertices and $L_{k} \leq X$, and

- $Z \leq X$ for some $Z \in \mathscr{M}(\tilde{G})$.

Lemma 2.37 For a digraph $G \in \mathscr{D}$ that has at least one loop in every weakly connected component, the set $\left\{G, G^{T}\right\}$ is definable. 
Proof We can still suppose that $G$ has more than one weakly independent components, for we have dealt with the other case previously. Let us consider a digraph $H \in \mathscr{O}(G)$. Since $H$ is weakly connected, the set $\tilde{H}$ is definable by Lemma 2.36 . Let $n(>1)$ be the number of weakly connected components of $G$ and $m$ be the number of its vertices. Let $H^{\prime}$ be the digraph that we get from $H$ by leaving the edges out that connect the "big circle" to the components of $G$, meaning we leave $n$ edges out and get $H^{\prime}=G \cup \dot{\cup} O_{m}$. $\tilde{H}^{\prime}$ contains exactly those digraphs $X$ such that

- $X$ is $n$ level under $Z$ for some $Z \in \tilde{H}$,

- there is no $Z \in \tilde{\sigma}_{m}$ for which $Z \leq X$, and

$-O_{m} \leq X$.

$H^{\prime}$ consists of $n+1$ weakly connected components from which exactly $n$ have loops in them, exactly the components corresponding to $G$. Finally $\tilde{G}$ is the set of those digraphs $X$ such that:

- $X \leq Z$ for some $Z \in \tilde{H}^{\prime}$,

- $X$ has $m$ vertices and is on the same level as $G$,

- $X$ has the same number of loops as $G$ has, and

- $Z \leq X$ for some $Z \in \mathscr{M}(\tilde{G})(\mathscr{M}(\tilde{G})$ is definable by Lemma 2.33$)$.

Theorem 2.38 For all $G \in \mathscr{D}$, the set $\left\{G, G^{T}\right\}$ is definable.

Proof We can still suppose $G$ has more than one weakly independent components for the same reason as above. Let us consider a digraph $H \in \mathscr{O}(G)$ again. By Lemma 2.36 the set $\tilde{H}$ is definable. We can define $\tilde{H}^{\prime}$ just as in the proof of Lemma 2.37. Let $G_{L}$ denote the digraph that consists of those weakly connected components of $G$ that contain a loop. By Lemma 2.37 the set $\tilde{G}_{L}$ is definable. $\tilde{G}$ is the set of those digraphs $X$ such that:

- $X$ has the same number of vertices and is on the same level as $G$,

- $X \leq Z$ for some $Z \in \tilde{H}^{\prime}$,

- $Z \leq X$ for some $Z \in \mathscr{M}(\tilde{G})$, and

$-Z \leq X$ for some $Z \in \tilde{G}_{L}$.

\section{The automorphism group of $(\mathscr{D}, \leq)$}

So far we know two automorphisms of $(\mathscr{D}, \leq)$, namely the trivial one and $G \mapsto$ $G^{T}$. In this section we prove that there is no other, meaning that the automorphism group of $(\mathscr{D}, \leq)$ is isomorphic to $\mathbb{Z}_{2}$.

Lemma 3.1 $G^{T} \leq G \cup \dot{\cup} O_{n}$ implies $G=G^{T}$ for every finite digraph $G$ and integer $2 \leq n$.

Proof Our first easy observation is that $X \leq O_{n}$ implies $X=X^{T}$. Let us denote the weakly connected components of $G$ by $\left\{G_{a}\right\}_{a \in A}$. Let $A=B \cup C$ such that $b \in B$ if 
and only if $G_{b}$ is embeddable into $O_{n}$. Now let us suppose that $G^{T} \leq G \dot{\cup} O_{n}$. With the notation just introduced

$$
\begin{gathered}
\left(\bigcup_{a \in A} G_{a}\right)^{T} \leq\left(\bigcup_{a \in A} G_{a}\right) \cup O_{n} \\
\bigcup_{a \in A} G_{a}^{T} \leq\left(\bigcup_{a \in A} G_{a}\right) \dot{\cup} O_{n} \\
\left(\bigcup_{b \in B} G_{b}^{T}\right) \cup\left(\bigcup_{c \in C} G_{c}^{T}\right) \leq\left(\bigcup_{b \in B} G_{b}\right) \cup\left(\bigcup_{c \in C} G_{c}\right) \cup O_{n}
\end{gathered}
$$

which obviously implies

$$
\bigcup_{c \in C} G_{c}^{T} \leq\left(\bigcup_{c \in C} G_{c}\right) \cup \underbrace{\left(\bigcup_{b \in B} G_{b}\right) \cup O_{n}}_{=: X} .
$$

If there exists a $c \in C$ for which $G_{c}^{T} \leq X$, then $G_{c}^{T} \leq O_{n}$, for $X$ consists of weakly connected components embeddable into $O_{n}$. Then, according to our first observation, $G_{c}^{T}=G_{c}$ which means $G_{c} \leq O_{n}$, a contradiction. This means there is no $c \in C$ for which $G_{c}^{T} \leq X$ so from (3) we deduce

$$
\begin{aligned}
& \bigcup_{c \in C}^{\cdot} G_{c}^{T} \leq \bigcup_{c \in C}^{\cdot} G_{c}, \text { and } \\
& \left(\bigcup_{c \in C}^{\cdot} G_{c}\right)^{T} \leq \bigcup_{c \in C} G_{c} .
\end{aligned}
$$

By transposing both sides the direction of the embeddability stays the same obviously, but we get the converse, implying

$$
\left(\bigcup_{c \in C} G_{c}\right)^{T}=\bigcup_{c \in C} G_{c}
$$

Using our first observation once more, we obtain

$$
\left(\bigcup_{b \in B} G_{b}\right)^{T}=\bigcup_{b \in B} G_{b}^{T}=\bigcup_{b \in B} G_{b}
$$

Finally, putting together what we have we get

$$
G=\bigcup_{a \in A} G_{a}=\left(\bigcup_{b \in B} G_{b}\right) \dot{\cup}\left(\bigcup_{c \in C} G_{c}\right)=\left(\bigcup_{b \in B} G_{b}\right)^{T} \dot{\cup}\left(\bigcup_{c \in C} G_{c}\right)^{T}=G^{T}
$$




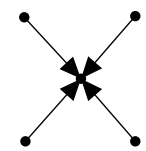

Fig. 13 The digraph $X_{4}$.

Definition 3.2 Let us call $X_{n}$ (see Fig. 13) the digraph with

$$
V\left(X_{n}\right)=\left\{v, v_{1}, v_{2}, \ldots, v_{n}\right\}, \quad E\left(X_{n}\right)=\left\{\left(v_{1}, v\right),\left(v_{2}, v\right), \ldots,\left(v_{n}, v\right)\right\} .
$$

Theorem 3.3 The poset $(\mathscr{D}, \leq)$ has exactly two automorphisms, namely the trivial and the one that maps every digraph to its transpose. Consequently, the automorphism group of $(\mathscr{D}, \leq)$ is isomorphic to $\mathbb{Z}_{2}$.

Proof It is easily seen that an automorphism can only move the elements of $\mathscr{D}$ inside definable sets, therefore from Theorem 2.38 it follows that it either does not move an element or maps it to its transpose. Let us consider an automorphism $\varphi: \mathscr{D} \mapsto \mathscr{D}$ for which there exists $G \in \mathscr{D}$ such that $G \neq G^{T}$ and $\varphi(G)=G$. We must show that $\varphi$ is the identity function. This can be done by showing that adding $G$ to the language of partially ordered sets as a constant results in every element of $\mathscr{D}$ becoming definable. So let us add $G$ to the language of partially ordered sets as a constant and pick an arbitrary $F \in \mathscr{D}$ that is not isomorphic to its transpose (those digraphs that are isomorphic to their transposes are definable by Theorem 2.38). Our goal will be to show that $F$ is definable.

Let $V(G)=\left\{v_{1}, v_{2}, \ldots, v_{n}\right\}$. Lemma 3.1 lets us define $G \cup O_{n+1}$ as the unique element from the definable set

$$
\left\{G \cup \dot{\cup} O_{n+1},\left(G \cup \dot{\cup} O_{n+1}\right)^{T}=G^{T} \dot{\cup} O_{n+1}\right\}
$$

that $G$ is embeddable into. Let us use the notation

$$
V\left(G \cup O_{n+1}\right)=V(G) \cup\left\{v_{1}^{\prime}, v_{2}^{\prime}, \ldots, v_{n+1}^{\prime}\right\} .
$$

We create a digraph $G^{\prime}$ (see Fig. 14) by adding edges to $G \cup O_{n+1}$ as follows:

$$
E\left(G^{\prime}\right)=E\left(G \cup O_{n+1}\right) \cup\left\{\left(v_{1}^{\prime}, v_{1}\right),\left(v_{2}^{\prime}, v_{1}\right),\left(v_{3}^{\prime}, v_{1}\right), \ldots,\left(v_{n+1}^{\prime}, v_{1}\right)\right\} .
$$

Now $G^{\prime}$ is definable as the unique element of the set $\left\{G^{\prime},\left(G^{\prime}\right)^{T}\right\}$ into which $G \dot{\cup}$ $O_{n+1}$ is embeddable. $X_{n+1}$ is the unique element from the set $\left\{X_{n+1},\left(X_{n+1}\right)^{T}\right\}$ that is embeddable into $G^{\prime}$ so it is definable too. $A$ is the unique element from the set $\left\{A, A^{T}\right\}$ that is embeddable into $X_{n+1}$. So far we have proven that $A$ is definable.

Now we do the same as above, but backwards. Let $m$ be the number of vertices of $F$. $X_{m+1}$ is the unique element in the set $\left\{X_{m+1},\left(X_{m+1}\right)^{T}\right\}$ that $A$ is embeddable into. Let $F^{\prime}$ be created from $F$ analogously to how $G^{\prime}$ was created from $G$ in (4) (see Fig. 14). Now $F^{\prime}$ is the only element from the set $\left\{F^{\prime},\left(F^{\prime}\right)^{T}\right\}$ that $X_{m+1}$ is embeddable into. Next, $F \cup O_{m+1}$ is the only element from the definable set

$$
\left\{F \cup \dot{O_{m+1}},\left(F \cup \dot{O_{m+1}}\right)^{T}=F^{T} \dot{\cup} O_{m+1}\right\}
$$




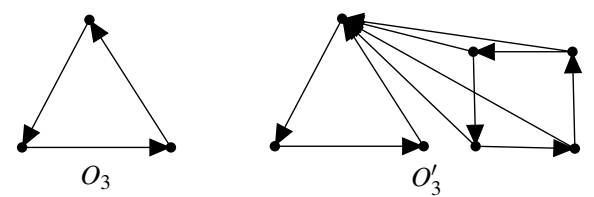

Fig. 14 The digraph $\mathrm{O}_{3}$ and a corresponding $\mathrm{O}_{3}^{\prime}$.

that is embeddable into $F^{\prime}$. Finally, by Lemma 3.1, $F$ is definable as the only element from the set $\left\{F, F^{T}\right\}$ that is embeddable into $F \dot{\cup} O_{m+1}$.

Acknowledgements The author is very thankful to his supervisor, Miklós Maróti, who introduced him to the problem investigated in this paper and supported him even from overseas.

\section{References}

1. J. Ježek and R. McKenzie, Definability in substructure orderings, I: finite semilattices, Algebra Universalis, 61, 2009, 59-75.

2. J. Ježek and R. McKenzie, Definability in substructure orderings, II: finite ordered sets, Order, 27, 2010, 115-145.

3. J. Ježek and R. McKenzie, Definability in substructure orderings, III: finite distributive lattices, Algebra Universalis, 61, 2009, 283-300.

4. J. Ježek and R. McKenzie, Definability in substructure orderings, IV: finite lattices, Algebra Universalis, 61, 2009, 301-312. 